\title{
Clinical study in diagnosis of placental implantation abnormality with transabdominal Doppler ultrasound
}

\author{
Fanwen Bu, Shuna Shen* \\ Third Affiliated Hospital of Inner Mongolia Medical University, Baotou, China
}

Received: January 7, 2017

DOI: $10.14725 /$ dcc.v4n1p5

\author{
Accepted: February 15, $2017 \quad$ Online Published: March 10, 2017 \\ URL: http://dx.doi.org/10.14725/dcc.v4n1p5
}

\begin{abstract}
Objective: To evaluate the ultrasonographic charateristics and the diagnostic value in placental implantation abnormality (PIA) with transabdominal Doppler ultrasound, and improve the rate of diagnosis accuracy in the prenatal and postnatal of PIA with transabdominal Doppler ultrasound, for early diagnosis and treatment, to avoid hysterectomy caused by fatal bleeding.

Methods: There were 61 cases complicated with PIA by operation and pathologically confirmed in our hospital. 33 cases were prenatal PIA, and 28 cases were postnatal PIA. The sonographic characteristics of PIA were summarized, the sensitivity and false negative rate of transabdominal Doppler ultrasound were analyzed with the results of surgical and pathological diagnosis. Results: In the images of prenatal PIA, the incidence of abundant blood flow signals in muscular layer of the placenta was the highest, which could reached $85.7 \%$, followed by placenta "vortex" and placenta previa; placental remnants were major sonographic performance in postnatal PIA, the incidence rate was $100 \%$, followed by abundant blood flow signals in muscular layer of the placenta and disappeared boundary between myometrium and placenta. 14 cases could be diagnosed as prenatal PIA accurately by transabdominal Doppler ultrasound, the sensitivity was $42.4 \%$, and the false negative rate was $57.6 \%$. 21 cases could be diagnosed as postnatal PIA accurately by transabdominal Doppler ultrasound, the sensitivity was $75.0 \%$, and the false negative rate was $25.0 \%$.

Conclusions: The specificity was low in prenatal PIA by transabdominal Doppler ultrasound, but the false negative rate was high. If necessary, the diagnostic accuracy could be improved combined with MRI. The specificity was much higher in postnatal PIA, and the false negative rate was low. The ultrasonography of PIA has some certain characteristics, we should strengthen the consciousness of the diagnosis in PIA, especially in the prenatal PIA, and improve the auxiliary diagnosis.
\end{abstract}

Key Words: Transabdominal Doppler ultrasound, Placental implantation abnormality, Ultrasonography, Plancental invasion, Missed diagnosis

In recent years, the WHO survey of maternal and child health in Asia have shown that the rate of cesarean section in our country is as high as $46.2 \%$. Among them, the rate of cesarean section without medical indication is $11.7 \% .^{[1]}$ The number of patients with cesarean section increased, and postoperative complications also increased sig- nificantly, such as postoperative adhesions and abdominal pain, uterine scar pregnancy and placenta previa. The incidence of placental implantation abnormality (PIA) is on the rise year by year. ${ }^{[2]}$ In the past 50 years, the incidence has increased 10 times, ${ }^{[3]}$ but the diagnosis rate is not high. Transabdominal color Doppler ultrasound is an important China.

\footnotetext{
*Correspondence: Shuna Shen; E-mail: shunashen@126.com; Address: Third Affiliated Hospital of Inner Mongolia Medical University, Baotou,
} 
auxiliary examination method in obstetrics and gynecology. The PIA cases confirmed by surgical pathology in our hospital from March 2009 to March 2014 were retrospectively analyzed, and the sonographic characteristics of PIA were summarized, and the reasons for missed diagnosis were analyzed.

\section{Data and methods}

\subsection{Data collection}

61 cases of PIA confirmed by surgery and pathology in our hospital from March 2009 to March 2014 were collected, of which 33 cases were prenatal PIA and 28 cases were postnatal PIA, patient age ranging from 23 to 44 years (mean = 36.4).

\subsection{Instruments and methods}

Siemens 512 and Philips IU22 high-end color Doppler ultrasound probe were used, convex array probe, the frequency of 3.5-5.5 MHz. The patients were in supine position during prenatal transabdominal ultrasound examination for a detailed show of fetus and appendage. The position of placenta, placental space, placental parenchyma, and demarcation of placenta and myometrium were detected by two-dimensional ultrasound, and the thickness of the myometrium at the placenta was measured. The color Doppler ultrasound imaging was used to investigate the signal display of the muscularis lateral flow in the placenta. The patients with suspected placenta previa should fill the bladder moderately before exploring the relationship between the placenta and the location of the cervix. Postnatal transabdominal color Doppler ultrasound: detection of placenta remnants, the thickness of the uterine myometrium at the placental attachment, and the boundary between the placenta and the myometrium. The presence of high velocity and low resistance arterial blood flow signals in the muscularis side of the placenta was measured by spectral Doppler ultrasound.

\subsection{PIA diagnostic criteria with transabdominal Doppler ultrasound ${ }^{[4]}$}

There were many different size and irregular liquid dark areas (placental "vortex") in the placenta, and Doppler ultrasound indicated turbulent flow. The myometrium in the rear of the placenta became thinner or disappearing. Placenta previa was located at the anterior wall of the uterus and behind the bladder. The placenta protruded to the bladder, and the interface between the bladder and the uterine wall was damaged. Doppler ultrasound showed that the blood vessels of the uterine serosa and bladder interface increased.

\subsection{Statistical analyses}

A retrospective analysis of 61 cases of PIA was performed by transabdominal color Doppler ultrasonography, and its characteristics were summed up and the incidence rate was calculated. The sensitivity and false negative rate of transabdominal color Doppler ultrasound were calculated with the results of surgical and pathological diagnosis.

\section{Results}

61 patients undergoing PIA surgery were enrolled in the biopsy, and the uterine smooth muscle was observed under the microscope. Among 33 cases of prenatal PIA, 14 cases were correctly diagnosed by transabdominal ultrasonography, the sensitivity was $42.4 \%$ and the false negative rate was $57.6 \%$, according to the pathological diagnosis results of PIA. The characteristics of the images were summarized: (1) Disappeared posterior placenta gap in 8 cases $(57.1 \%)$. No echo vessels, weak musculature and hyperechoic decidua interface appeared behind the placenta, no echo area disappeared when placenta accreted. (2) Placental "vortex" in 11 cases (78.6\%). Abnormal increase of sinusoids in the placenta, different sizes of sonography, irregular anechoic area, and weak echoes in the placenta appeared. This phenomenon is caused by the placenta invading the muscular arterioles, high-speed arterial blood flowing into the placenta sinusoid and generating cloud-like roll. (3) Thinning muscular layer of the placenta in 6 cases $(42.9 \%)$. Even muscular echo disappeared. (4) Placenta previa in 9 cases (64.3\%). (5) Abundant blood flow signals in muscular layer of the placenta in 12 cases $(85.7 \%)$. Among 28 cases of postnatal PIA, 21 cases were correctly diagnosed by transabdominal ultrasonography, the sensitivity was $75 \%$ and the false negative rate was $25 \%$. The characteristics of the images were summarized: (1) Placental remnants in 21 cases (100\%). The hyperechoic masses of the intrauterine inhomogeneity were close to the wall of the uterus. (2) The boundary between the placenta and the myometrium disappeared in 12 cases $(57.1 \%)$. (3) Pulsed Doppler spectrum showed the visible high-speed low resistance artery blood flow signals in muscular layer of the placenta in 7 cases (33.3\%). (4) The muscular layer of the placenta was thinning or weak echo disappeared in 19 cases $(90.5 \%$ ) (see Table 1$)$.

\section{Discussion}

There are many pathogenic factors that contribute to the development of PIA. Fan JM et al. ${ }^{[5]}$ reported that the risk factors leading to PIA included the history of miscarriage, placenta previa and pregnancy complicated with hypertension. Independent risk factors for PIA were placenta previa, pregnancy-associated hypertension and education. Fitzpatrick et al. ${ }^{[6]}$ confirmed that advanced age was a risk fac- 
tor of PIA for pregnant women. Sinha et al. ${ }^{[7]}$ proposed that PIA was related with production times, previous history of cesarean section and history of abortion. The incidence increased with the age of pregnant women, production times as well as the number of cesarean section. Most of the cases in this study resulted from one or more of the above factors.

Table 1: Prenatal and postnatal PIA sonographic performance comparison [n (\%)]

\begin{tabular}{ll}
\hline Prenatal sonographic performance & Incidence rate \\
\hline Disappearance of posterior placenta gap & $8(57.1)$ \\
Placenta "vortex" & $11(78.6)$ \\
Placental muscle thinning & $6(42.9)$ \\
Placenta previa & $9(64.3)$ \\
$\begin{array}{l}\text { Abundant signals of blood flow in muscular } \\
\text { layer of the placenta }\end{array}$ & $12(85.7)$ \\
\hline Postnatal sonographic performance & Incidence rate \\
\hline $\begin{array}{l}\text { Placental residue } \\
\text { Disappearance of boundary between muscular } \\
\text { layer and placenta }\end{array}$ & $21(100)$ \\
$\begin{array}{l}\text { Placental muscular layer weakness } \\
\text { Abundant signals of blood flow in muscular }\end{array}$ & $7(33.3)$ \\
layer of the placenta & $19(90.5)$ \\
\hline
\end{tabular}

Among the five manifestations of prenatal PIA, the incidence of abundant placental blood flow signals, that is, prominent placental vasodilatation, is the highest, followed by placenta "vortex". The placental vortex means the blood pool in the placental parenchyma presents a high speed, low resistance and high perfusion type of bright arterial blood flow signal, which is like the "Fire Sea" sign. It is consistent with the ultrasonic diagnostic performance of placental lacuna blood flow reported by Meng XY. ${ }^{[8]}$ Wang YL et al. ${ }^{[9]}$ found that the prenatal placenta "vortex" sign had a specific diagnostic value. We believe that using color Doppler flow imaging to detect blood flow in the myometrium of placenta and placenta is very important for improving the detection rate of transabdominal color Doppler ultrasound in PIA. If combined with power Doppler ultrasound, it can show lower flow velocity in the placental blood vessel network, and this function is not limited by Doppler angle, the blood flow of PIA shows more advantages. Among 14 cases of prenatal PIA accurately diagnosed by transabdominal color Doppler ultrasound, placenta previa was found in 9 cases, and 4 of them were central placenta previa. PIA has the same pathogenic mechanism with placenta previa: the underlying decidua is poorly developed, and the blood circulation is insufficient. In order to better exchange the nutrients with the placental blood vessels, the villi intrude into the myometrium and lead to PIA. The incidence of the thinning of myometrium at the placenta in prenatal PIA was $42.9 \%$. The reason for the low incidence is that the junction of normal uterus and bladder wall is a smooth hyperechoic line. When PIA occurs, the hyperechoic line is raised or interrupted, resulting in a deviation of the thickness of the my- ometrium. The anechoic region was thinner or disappearing. In fact, the image shows that the anechoic zone thinning or disappearing does not occur in a large area, but a partial inhomogeneous thinning. For mild and moderate PIA, there are differences in the subjective judgment of different ultrasound doctors. Placental remnants are the primary image of postnatal PIA, followed by the abundant blood flow signal in the placental layer and the disappearance of the boundary between the placenta and the myometrium. Placental remnants are found in all cases of postnatal PIA diagnosis, and the placenta is not easy to peel off. There was no "vortex" structure and blood flow signal in the placenta remnants. The boundary between the local myometrium and the placenta remnants in PIA is not clear. Doppler flow spectrum showed low resistance and high nutrient artery perfusion flow, placental blood flow signals of the myometrium side showed bright festoons performance. The invasion of the muscularis in placental firstly shows an unclear boundary between the remaining placental hyperechoic and hypoechoic myometrium, which is intermingled with vascular network. In addition, the structure of the infringing hypoechoic muscularis and the recognized thinning of the same uterus layer in the same level can indicate PIA.

In this study, 19 cases of missed diagnosis of prenatal PIA were diagnosed by transabdominal ultrasonography. 17 cases of the placenta were located in the posterior wall of the uterus and the gestational ages are relatively large, so that it is more difficult to display the placenta by transabdominal color Doppler ultrasound. In addition, in the third trimester of pregnancy, normal myometrium is very thin, and it is difficult to determine the extent of the placenta invaded the myometrium. In 2 cases, the PIA was not significant in the first trimester of pregnancy when ultrasound examination was performed. The placenta implanted myometrium was relatively shallow. It was diagnosed as PIA after operation. These two patients have multiple histories of dilatation and curettage. In this study, the rate of missed diagnosis of prenatal PIA by transabdominal ultrasonography was high, which was consistent with the data reported in other literatures. The rate of missed diagnosis was $68 \%$ reported by the domestic scholar $\mathrm{Hu}$ BQ et al. ${ }^{[10]}$ and $61.6 \%$ by Teng et al. ${ }^{[1]}$ There are two reasons that accounts for the high rate of missed diagnosis of prenatal PIA. First, the accurate diagnosis of PIA is affected by the placental position factor, and the posterior wall placenta is not easy to show because of the occlusion of the fetal body. Secondly, the domestic research on the diagnosis of PIA during pregnancy is not enough. ${ }^{[12]} 7$ cases of postnatal PIA were missed by transabdominal color Doppler ultrasound. The reasons of missed diagnosis are: (1) Ultrasound doctors pay more attention to the diagnosis of placental remnants and ignore the PIA. (2) The clinical features of postnatal PIA are less, and the doctors can not consider the problem in combination with clinical manifestations. (3) There is no specific sign of the placental implantation, and it is difficult to determine PIA. 
The depth of the infiltrating layer of the placenta is not accurately determined by transabdominal color Doppler ultrasound, no matter in prenatal PIA or postnatal PIA, but the thickness of the placental layer can be measured. Compared with the ultrasound examination, MRI has a significant advantage in determining the depth of PIA myometrium infiltration. MRI can accurately show the location and depth of placenta infiltrating myometrium before birth, and the coincidence rate with pathological diagnosis after cesarean section is high. ${ }^{[11,12]}$ It can further compensate for the low sensitivity of prenatal PIA diagnosis by transabdominal color Doppler ultrasound, especially the placenta locates on the posterior wall of uterus. However, MRI is costly and not widely available in small-scale medical institutions. Therefore, compared with MRI, transabdominal ultrasonography can be used as the preferred imaging method to further perform MRI in patients suspected of having PIA. It is reported that ultrasound combined with MRI diagnosis of PIA carries the highest sensitivity. ${ }^{[13]}$ Right cesarean section pregnancy with placenta previa should be routinely examined by transabdominal ultrasonography combined with MRI. At present, the three-dimensional transabdominal color Doppler ultrasound technology is developing rapidly. Three-dimensional color Doppler ultrasound can display blood vessel branching images. The diagnostic sensitivity is $97 \%$ and the specificity is $92 \%$. However, three-dimensional image post-processing will produce artifact, and the practical application of threedimensional ultrasound is limited. ${ }^{[14]}$

\section{References}

[1] Pisake L, Malinee L, A Metin G, et al. Method of delivery and pregnancy outeomeos in Asia: the WHO global survey on maternal and perinatal health 2007-2008. Lancet. 2010; 375(9713): 490-499. https://doi .org/10.1016/S0140-6736(09)61870-5

[2] Li J. Clinical analysis of three cases of placenta previa and placenta implantation. Clinical Misdiagnosis \& Mistherapy. 2013; 26(9): 2426.

[3] Wu Y. Value analysis of ultrasonic diagnosis of placental implantation in late pregnancy. Medical Innovation of China. 2013(7): 8-79.

[4] Deng XD. Prenatal ultrasound diagnosis and differential diagnosis. Beijing: People's Military Medical Publishing House; 2014. 292$293 \mathrm{p}$.

[5] Fan JM, Ren JF. Analysis of risk factors for 65 patients with placental implantation. Current Advances in Obstetrics and Gynecology. 2013; 22(5): 401-404.

[6] Fitzpatrick KE, Sellers S, Spark P, et al. Incidence and risk factors for placenta accrete/increta/percreta in the UK: a national casecontrol study. Plos One. 2012; 7(12): e52893. PMid: 23300807. https://doi.org/10.1371/journal.pone.0052893

[7] Sinha P, Pniya O, Bewley S. Coping with placenta previa and accreta in a DGH setting and words of caution. J Obstet Gynaecol. 2005(25): 334-338. PMid: 16091311. https://doi.org/10. 1080/01443610500119739

[8] Meng XY, Xie LM. Application and evaluation of ultrasound and MRI in the diagnosis of placenta implantation. Chinese Journal of Interventional Imaging and Therapy. 2013; 10(1) : 438-441.

\section{Conclusions}

Placenta position and gestational age are the important factors influencing the detection of prenatal PIA. The depth of placenta accreta and the understanding of PIA by ultrasound doctors are important factors that affect postnatal PIA detection. An effective way to reduce the missed diagnosis of prenatal and postnatal PIA is to apply low-velocity blood flow signals in the placental vascular network by using energy Doppler ultrasound in patients with high-risk recurrent vaginal bleeding during pregnancy to increase the sensitivity of blood flow signals and reduce missed diagnosis. Application of three-dimensional ultrasound combined with two-dimensional ultrasound, PIA diagnostic accuracy will be higher than the application of two-dimensional ultrasound alone. ${ }^{[15]}$ The incidence of placenta previa in the middle and late phrase of pregnant women with the history of cesarean section is high, and there is a risk of rupture of uterus and fatal postnatal hemorrhage. The diagnosis of PIA by transabdominal ultrasonography is of great significance. PIA sonographic performance has certain characteristics. Ultrasound doctors should strengthen the diagnosis of PIA awareness, especially in prenatal PIA, carefully explore the sonographic performance, and improve auxiliary diagnosis for PIA.

\section{Conflicts of Interest Disclosure}

The authors have no conflicts of interest related to this article.

[9] Wang YL, LiuY, Zhang LP, et al. Application of color Doppler ultrasound in the diagnosis of placental implantation. Chinese Journal of Coal Industry Medicine. 2013; 16(1): 37-39.

[10] Hu BQ, Wu JX, Yang JH. Prenatal ultrasound diagnosis of placenta implantation. Youjiang Medical Journal. 2013; 41(6): 889-890.

[11] Teng X, Guo X, Zhu QR, et al. Ultrasonographic analysis of placental implantation. Chinese Imaging Journal of Integrated Traditional and Western Medicine. 2013; 11(4): 425-426.

[12] Ling RN, Zhao YH, Zhu Jin, et al. MRI analysis of placental implantation. Guangdong Medical Journal. 2014; 35(20): 3205-3207.

[13] Bai GH, Jiang X, Chen Yu, et al. Comparative study of diagnostic value of magnetic resonance and ultrasound in placental implantation. Journal of Medical Research. 2012; 41(1): 96-100.

[14] Jiang KM, Li ST, Zhong Xi, et al. Prenatal MRI expression and pathological basis of placental implantation. Chinese Journal of Medical Imaging Technology. 2013(29): 1002-1005.

[15] Chen WS, Li QS, Zhang JT, et al. Diagnostic value of color Doppler ultrasound, MRI and two methods of combined test on placenta implantation. Chinese Journal of Ultrasound in Medicine. 2010(26): 1116-1118.

[16] Shih JC, Jaraquemada JMP, Su YN, et al. Role of three-dimensional power Doppler in the antenatal diagnosis of plancenta accrete: Comparison with gray-scaleand color Doppler techniques. Ultrasound Obstet Gynecol. 2009; 33(2): 193-203. PMid: 19173239. https: //doi.org/10.1002/uog. 6284

[17] Li XG, Li YM. Clinical application of two-dimensional combined three-dimensional ultrasound diagnosis of placenta implantation. Medical Journal of West China. 2010(22): 1925-1926.

[18] Yang HX. A case of cicatricial pregnancy associated with placenta previa and placenta implantation after cesarean section. Chinese Journal for Clinicians. 2014; 42(6): 564. 